Research Article

\title{
A New Method for Reliability-Based Sensitivity Analysis of Dynamic Random Systems
}

\author{
Qianqian Wang $\mathbb{D}$, Changchun Wang $(\mathbb{D}$, and Hui Wang $(\mathbb{D}$ \\ School of Mechanical Electronic and Vehicle Engineering, WeiFang University, Weifang 261061, China \\ Correspondence should be addressed to Qianqian Wang; wqq@wfu.edu.cn
}

Received 28 April 2019; Revised 7 July 2019; Accepted 1 August 2019; Published 11 September 2019

Academic Editor: Filippo Cacace

Copyright (C) 2019 Qianqian Wang et al. This is an open access article distributed under the Creative Commons Attribution License, which permits unrestricted use, distribution, and reproduction in any medium, provided the original work is properly cited.

\begin{abstract}
A novel numerical method for investigating time-dependent reliability and sensitivity issues of dynamic systems is proposed, which involves random structure parameters and is subjected to stochastic process excitation simultaneously. The Karhunen-Loève (K-L) random process expansion method is used to express the excitation process in the form of a series of deterministic functions of time multiplied by independent zero-mean standard random quantities, and the discrete points are made to be the same as Legendre integration points. Then, the precise Gauss-Legendre integration is used to solve the oscillation differential functions. Considering the independent relationship of the structural random parameters and the parameters of random process, the time-varying moments of the response are evaluated by the point estimate method. Combining with the fourth-moment method theory of reliability analysis, the dynamic reliability response can be evaluated. The dynamic reliability curve is useful for getting the weakness time so as to avoid breakage. Reliability-based sensitivity analysis gives the importance sort of the distribution parameters, which is useful for increasing system reliability. The result obtained by the proposed method is accurate enough compared with that obtained by the Monte Carlo simulation (MCS) method.
\end{abstract}

\section{Introduction}

Random analysis of vibrating structures has received much more attention in recent years [1-4]. Mechanical structures always contain unavoidable uncertain factors such as geometrical errors, uncertainty of material properties, and manufacturing errors, which constitute the basic random parameters of the vibrating system. And the structures may be subjected to stochastic process excitation in the meanwhile, such as the excitation from the base, ocean wave, wind, and rough road. If the responses of the structures under random conditions exceed the allowable boundary, it will cause failures, e.g., base vibration, noise, and interference. The reliability analysis of random vibrating systems aims to analyze failure probability in the vibrating process especially at the unstable time when the machine is started. The reliability based-sensitivity analysis ranks the importance of the distribution characteristics of the random parameters contributing to reliability, which is of great significance for increasing the reliability value.
For the time-independent random systems, traditional methodologies can be classified into two categories: the numerical Monte Carlo simulation method [5] and analytical reliability methods (e.g., the first-order reliability method, the second-order reliability method, and the advanced mean value method). However, the state functions of complex vibrating structures are always in the implicit form and difficult to obtain. In these conditions, the analytical reliability methods are seldom applicable. Therefore, more suitable methods are developed for implicit limit-state functions, e.g., the response surface method [6-9], the artificial neutral network method [10], and the point estimate method $[11,12]$. Within all these methods, the point estimate method is convenient for implicit multivariate state functions [13-15].

As the exciting force of the dynamic random system is a time-dependent random process, it is computationally expensive to obtain the time-varying reliability value in the time domain. $\mathrm{Hu}[16,17]$ used an extreme value response method to solve the time-dependent reliability problems, 
and Wang et al. [18] used this method in kinematic reliability analysis. This extreme value method transforms the time-dependent problems to time-independent problems and simplifies the calculation. However, it ignores random response at each time point. Another method is decomposing the spectral representation of the random process using K-L expansion $[19,20]$, orthogonal series expansion, expansion optimal linear estimation, etc. Of all these expansion methods, K-L expansion is an efficient tool for the excitation process in the dynamic system.

In the past literatures, a number of works [21, 22] have been performed on linear structures' reliability analysis issues. The perturbation method, fourth-moment method combined with the Edgeworth series [23], improved response surface method combined with the trigonometric series expression of the stochastic process $[24,25]$, path integration method $[26,27]$, and a new probability density evolution method [2] for dynamic response analysis and reliability assessment were investigated in recent years. However, time-varying random responses of structures with random structure parameters and random process excitation are not properly investigated in all the literatures mentioned above.

In the present study, an attempt has been made to develop a time-varying reliability and sensitivity analysis for a random parametric structural system under stationary or nonstationary excitation incorporating uncertainty in the structural parameters. An approach for evaluating the response of the random vibration is proposed by combining K-L expansion, Gauss-Legendre precise integration, and the dimension reduction point estimate method. And then, the reliability-based sensitivities with respect to the mean and variance of the random structural parameters are analyzed based on the system reliability utilizing the core function method. The process of reliability and sensitivity analysis of the random dynamic system is presented in Figure 1. The main idea of the process is as follows:

(1) The Gaussian process is expanded by the K-L method to a series of time-dependent deterministic functions multiplied by Gaussian random parameters, which are dispersed at the Gauss-Legendre integral points.

(2) The precise integration is utilized to solve the differential equations of the random structures subjected to each expansion deterministic function.

(3) Combining with the point estimate method, the moments of each response under the series of deterministic functions are calculated.

(4) Considering the Gaussian random parameters produced in the K-L expansion, the moments of the structure's random response are calculated.

(5) The moment method is used to estimate the reliability and sensitivities.

\section{K-L Expansion of the Stochastic Process}

A quite general and precise spectral representation utilized for the Gaussian random process is the K-L expansion of the covariance function. This expansion is available to stationary and nonstationary stochastic processes. The expansion of a stochastic process takes the following form:

$$
f(t)=f^{(0)}(t)+\sum_{j=1}^{\infty} \xi_{j} f^{(j)}(t),
$$

where the first term $f^{(0)}(t)$ is the mean of the random process. The second term is the summation of a set of orthogonal zero-mean random variables $(\xi)$ with unit variance multiplied by a series of deterministic functions of time:

$$
\begin{aligned}
E\left[\xi_{j}\right] & =0, \\
E\left[\xi_{i} \xi_{j}\right] & =\delta_{i j},
\end{aligned}
$$

where $\delta_{i j}$ denotes Kronecker's delta symbol. In practical applications, the continuous stochastic process $f(t)$ is dispersed at time points $t_{k}=k \Delta t, k=1,2, \ldots, n$, and the $n \times n$-dimensional covariance matrix $C_{f f}\left(t_{k}, t_{k}^{\prime}\right)$ of the dispersed stochastic process is obtained:

$$
C_{f f}=E\left\{\left[f\left(t_{k}\right)-f^{(0)}\left(t_{k}\right)\right]\left[f\left(t_{k}^{\prime}\right)-f^{(0)}\left(t_{k}^{\prime}\right)\right]^{T}\right\} .
$$

The dispersed values $\left\{f^{(j)}\left(t_{k}\right)\right\}_{k=1}^{L}$ of the deterministic functions $f^{(j)}(t)$ in the K-L expansion terms are uniquely specified by the eigenvectors $\varphi_{j}$ and eigenvalues $\lambda_{j}$ of the symmetric covariance matrix $\mathbf{C}=\left[C_{i k}\right]$ as follows:

$$
f^{(j)}\left(t_{k}\right)=\phi_{k j} \sqrt{\lambda_{j}},
$$

where $\phi_{k j}$ denotes the $k$ th component of the $j$ th eigenvector. When the expansion term equals the number of time points $n$, it is accurate for the K-L representation to describe the stochastic process. However, in practical applications, it is too complex for all terms to be considered. Assuming the eigenvalues are decreasingly ordered, and accepting a tolerance $\varepsilon$, the number $N<n$ can be estimated accordingly. And then, equation (1) can be written as

$$
\widehat{f}\left(t_{k}\right)=f^{(0)}\left(t_{k}\right)+\sum_{j=1}^{N} \xi_{j} f^{(j)}\left(t_{k}\right) .
$$

If the dispersed time points $t_{k}$ are replaced by the Gauss-Legendre integration points as described in Section 3, the dispersed exciting force functions can be obtained by equation (5), and they are applicable in the following precise integration.

To quantify the accuracy of the truncated K-L expansion with number $N$, the following variance and covariance errors are defined according to literatures $[28,29]$ : 


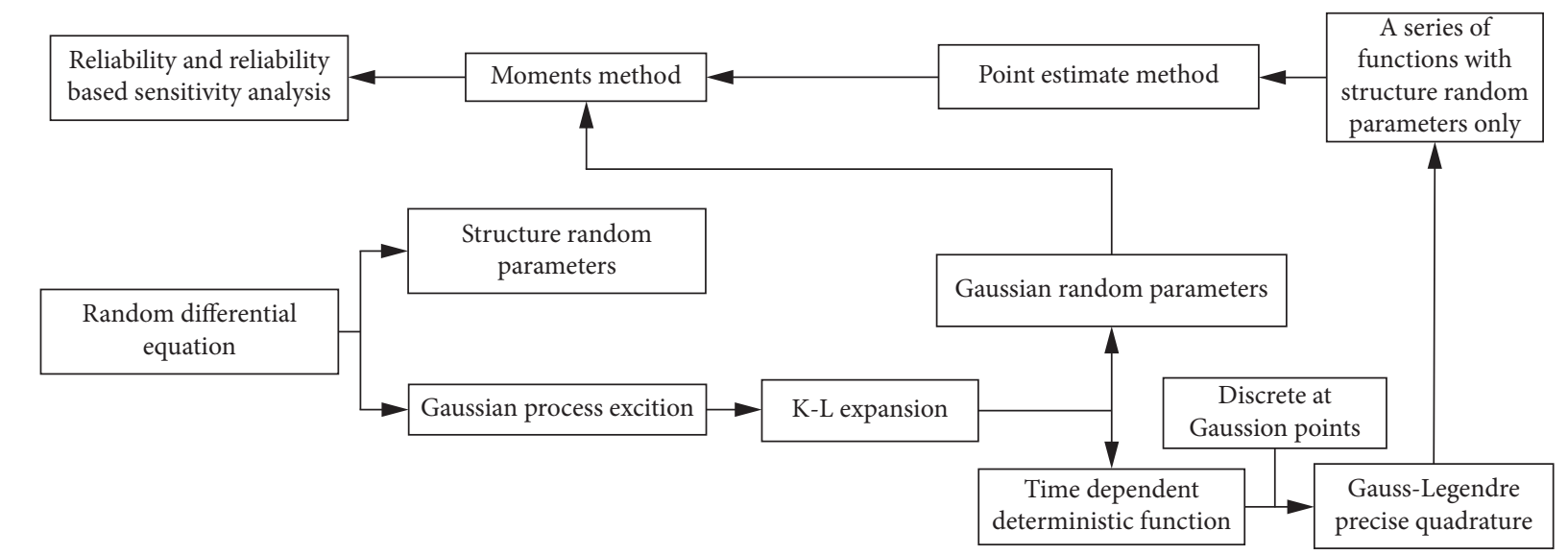

FIGURE 1: Flow diagram of the solution process.

$$
\begin{aligned}
\varepsilon_{\mathrm{Var}, N}(t) & =\frac{\operatorname{Var}[f(t)-\widehat{f}(t)]}{\operatorname{Var}[f(t)]}=\frac{\sigma^{2}(t)-\sum_{j=1}^{N} \lambda_{j} \phi_{j}^{2}(t)}{\sigma^{2}(t)}, \\
\varepsilon_{\operatorname{Cov}, N}\left(t, t^{\prime}\right) & =\frac{\left|\operatorname{Cov}\left[f^{\prime}(t), f^{\prime}\left(t^{\prime}\right)\right]-\operatorname{Cov}\left[\widehat{f}(t), \hat{f}^{\prime}\left(t^{\prime}\right)\right]\right|}{\operatorname{Cov}\left[f^{\prime}(t)-f^{\prime}\left(t^{\prime}\right)\right]},
\end{aligned}
$$

where $\sigma^{2}(t)$ is the variance of the random process and Cov [.] is the covariance function. $f^{\prime}(t)=f(t)-f^{(0)}(t)$ denotes fluctuations around the mean value.

\section{Solution of Random Vibration}

3.1. Precise Gauss-Legendre Integration Points [30-33]. A multi-degree-of-freedom linear random vibration system can be expressed by differential equations in the matrix form as follows:

$$
\begin{aligned}
\mathbf{M Y}+\mathbf{K Y}+\mathbf{C Y} & =\mathbf{f}(t), \\
\left.\mathbf{Y}(t)\right|_{t=0} & =\dot{\mathbf{Y}}\left(t_{0}\right), \\
\left.\dot{\mathbf{Y}}(t)\right|_{t=0} & =\ddot{\mathbf{Y}}\left(t_{0}\right),
\end{aligned}
$$

where $\mathbf{M}, \mathbf{C}$, and $\mathbf{K}$ are the mass, damping, and stiffness matrices, respectively; $\mathbf{Y}(t), \dot{\mathbf{Y}}(t)$, and $\ddot{\mathbf{Y}}(t)$ are the displacement, velocity, and acceleration vectors, respectively; and $\mathbf{f}(t)$ is the force vector.

The state equation for the random vibration system can be written as

$$
\dot{\mathbf{v}}=\mathbf{H v}+\mathbf{F}(t)
$$

where

$$
\begin{aligned}
\mathbf{v} & =\left[\begin{array}{c}
\mathbf{Y} \\
\dot{\mathbf{Y}}
\end{array}\right], \\
\mathbf{H} & =\left[\begin{array}{cc}
\mathbf{0} & \mathbf{I} \\
-\mathbf{M}^{-1} \mathbf{K} & -\mathbf{M}^{-1} \mathbf{C}
\end{array}\right], \\
\mathbf{F}(t) & =\left[\begin{array}{c}
0 \\
\mathbf{M}^{-1} \mathbf{f}(t)
\end{array}\right] .
\end{aligned}
$$

The general solution of equation (7) is

$$
\mathbf{v}(t)=\exp (t \mathbf{H}) \mathbf{v}_{0}+\int_{0}^{t} \exp ((t-\tau) \mathbf{H}) \mathbf{F}(\tau) d \tau
$$

where $\mathbf{v}_{0}$ is the initial value of the dynamic system.

The interested time interval of the structural dynamic response is discredited at time points $t_{k}=k \Delta t, k=1,2, \ldots, q$, as described in Section 2. The responses at time $t_{k-1}$ and $t_{k}$ are written as

$$
\mathbf{v}\left(t_{k-1}\right)=\exp \left(t_{k-1} \mathbf{H}\right) \mathbf{v}_{0}+\int_{0}^{t_{k-1}} \exp \left(\left(t_{k-1}-\tau\right) \mathbf{H}\right) \mathbf{F}(\tau) d \tau,
$$

$$
\mathbf{v}\left(t_{k}\right)=\exp \left(t_{k} \mathbf{H}\right) \mathbf{v}_{0}+\int_{0}^{t_{k}} \exp \left(\left(t_{k}-\tau\right) \mathbf{H}\right) \mathbf{F}(\tau) d \tau
$$

Noting that $t_{k}=t_{k-1}+\Delta t$, the relationship between $\mathbf{v}\left(t_{k-1}\right)$ and $\mathbf{v}\left(t_{k}\right)$ obtained from equations (12) and (13) is

$$
\mathbf{v}\left(t_{k}\right)=\mathbf{T} \mathbf{v}_{k-1}+\int_{t_{k-1}}^{t_{k}} \exp \left(\left(t_{k}-\tau\right) \mathbf{H}\right) \mathbf{F}(\tau) d \tau,
$$

where $\mathbf{T}=\exp (\mathbf{H} \Delta t)$, and the accurate evaluation of $\mathbf{T}$ can be deduced from the following equation:

$$
\mathbf{T}=\exp (\mathbf{H} \Delta t)=[\exp (\mathbf{H} \tau)]^{2^{m}},
$$

in which $\tau=\Delta t / 2^{m}$. Expanding $\exp (\mathbf{H} \tau)$ by Taylor series, the following expression is obtained:

$$
\exp (\mathbf{H} \tau) \approx \mathbf{I}+\mathbf{H} \tau+\frac{(\mathbf{H} \tau)^{2}}{2}=\mathbf{I}+\mathbf{T}_{0},
$$

in which $\mathbf{T}_{0}=(\mathbf{H} \tau) \cdot[\mathbf{I}+(\mathbf{H} \tau / 2)]$ and $\mathbf{I}$ is the unit matrix.

It is further noted that

$$
\begin{gathered}
{[\exp (\mathbf{H} \tau)]^{2}=\left(\mathbf{I}+\mathbf{T}_{0}\right)^{2}=\mathbf{I}^{2}+2 \mathbf{I} \cdot \mathbf{T}_{0}+\mathbf{T}_{0}^{2}=\mathbf{I}+\mathbf{T}_{1},} \\
{[\exp (\mathbf{H} \tau)]^{2^{2}}=\left(\mathbf{I}+\mathbf{T}_{1}\right)^{2}=\mathbf{I}^{2}+2 \mathbf{I} \cdot \mathbf{T}_{1}+\mathbf{T}_{1}^{2}=\mathbf{I}+\mathbf{T}_{2},} \\
\ldots \quad \ldots \quad \ldots \\
\ldots \quad \ldots \quad \mathbf{I}_{w-1}^{2}+\mathbf{T}_{w-1}^{2}=\mathbf{I}+\mathbf{T}_{w} .
\end{gathered}
$$


So the recurrence formula is concluded as

$$
\mathbf{T}_{w}=2 \mathbf{T}_{w-1}+\mathbf{T}_{w-1} \cdot \mathbf{T}_{w-1}, \quad w=1,2, \ldots, m,
$$

and the solution of $\mathbf{T}$ can be deduced using the recurrence formula.

The random load process vector $\mathbf{F}(t)$ in equations (9)-(14) is represented by K-L expansion as

$$
\mathbf{F}(t)=\left\{\mathbf{M}^{-1}\left[\mathbf{f}^{(0)}(t)+\sum_{j=1}^{N} \xi_{j} \mathbf{f}^{(j)}(t)\right]\right\} .
$$

Therefore, the $j$ th term of $\mathbf{F}(t)$ and its corresponding solution $\mathbf{v}_{k}^{(j)}$ at time $t_{k}$ can be written, respectively, as

$$
\begin{aligned}
\mathbf{F}^{(j)}(t)= & \left\{\begin{array}{c}
\mathbf{0} \\
\mathbf{M}^{-1} \mathbf{f}^{(j)}(t)
\end{array}\right\}, \\
\mathbf{v}_{k}^{(j)}= & \mathbf{T}_{k-1}^{(j)}+\int_{t_{k-1}}^{t_{k}} \exp \left(\left(t_{k}-\tau\right) \mathbf{H}\right) \mathbf{F}^{(j)} \\
& \cdot(\tau) d \tau, \quad j=1, \ldots, N .
\end{aligned}
$$

The integration of equation (21) can be calculated using the Gauss-Legendre numerical method, and the solution can be represented as

$$
\begin{aligned}
\mathbf{v}_{k}^{(j)}= & \mathbf{T}_{k-1}^{(j)}+\frac{\Delta t}{2} \sum_{l=1}^{M} w_{l} \mathrm{e}^{\mathbf{H}\left[\left(\Delta t\left(1-\zeta_{l}\right)\right) / 2\right]} \\
& \cdot \mathbf{F}^{(j)}\left(t_{k-1}+\Delta t \frac{1+\zeta_{l}}{2}\right),
\end{aligned}
$$

where $M$ is the number of Gaussian integral points and $\zeta_{l}$ and $w_{l}$ are the Gaussian integral point and the corresponding weight, respectively.

Furthermore, for the linear systems, as $\mathbf{v}_{k}^{(j)}=\left[\mathbf{Y}_{k}^{(j)}\right.$, $\left.\dot{\mathbf{Y}}_{k}^{(j)}\right]^{T}$, the response $\mathbf{Y}$ of the random vibrating structure can be expressed as

$$
\mathbf{Y}_{k}=\mathbf{Y}_{k}^{(0)}+\sum_{j=1}^{N} \xi_{j} \mathbf{Y}_{k}^{(j)}
$$

3.2. Statistical Moment of the Random Extreme Response by Dimension Reduction Point Estimate Method. In each term of random response $\mathbf{Y}^{(\mathrm{j})}(j=0,1, \ldots, N)$, the structural random parameters in vector $\mathbf{X}$ are time independent. Therefore, it is suitable to use the dimension reduction point estimate method to evaluate the moments of the $j$ th response. The correlated nonnormal random parameters can be transformed to independent standard normal variables by the inverse normal transformation, which can be written as

$$
\mathbf{X}=T^{-1}(\mathbf{U})
$$

in which $\mathbf{U}$ is the $n$-dimensional independent standard normal vector and $T^{-1}(\cdot)$ is the inverse transforming function such as the Rosenblatt transformation, Nataf transformation, and third-moment transformation technique, which is determined by the known conditions [34].

Then, the dimension reduction method at time $t_{k}$ is expressed as

$$
\widehat{\mathbf{Y}}_{k}^{(j)}(\mathbf{X})=\sum_{r=1}^{n} \mathbf{Y}_{k}^{(j)}\left[T^{-1}\left(\mathbf{U}_{r}\right)\right]-(n-1) \mathbf{Y}_{k}^{(j)}(\boldsymbol{\mu})
$$

where $\mu$ represents the vector of mean values of the random variables, and

$$
\mathbf{U}_{r}=\left[u_{\mu 1}, u_{\mu 2}, \ldots, u_{\mu r-1}, u_{r}, u_{\mu r+1}, \ldots, u_{\mu n}\right],
$$

in which $u_{r}$ is the only standard normal distributed random parameter and the other variables are set equal to mean values transformed into the standard normal space.

Considering $\mathbf{Y}_{k}^{(j)}\left[T^{-1}\left(\mathbf{U}_{r}\right)\right]=\mathbf{Y}_{k, r}^{(j)}$, the $l$ th origin moment of the $j$ th random response can be expressed by the binomial theorem as

$$
\begin{aligned}
E\left\{\left[\widehat{\mathbf{Y}}_{k}^{(j)}\right]^{l}\right\} & =E\left\{\left[\sum_{r=1}^{n} \mathbf{Y}_{k, r}^{(j)}-(n-1) \mathbf{Y}_{k}^{(j)}(\boldsymbol{\mu})\right]^{l}\right\} \\
& =E\left\{\left[\sum_{s=1}^{l} C_{l}^{s}\left[\sum_{r=1}^{n} \mathbf{Y}_{k, r}^{(j)}\right]^{s}\left[-(n-1) \mathbf{Y}_{k}^{(j)}(\boldsymbol{\mu})\right]\right]^{l-s}\right\} \\
& =\sum_{s=1}^{l} C_{l}^{s} E\left\{\left[\sum_{r=1}^{n} \mathbf{Y}_{k, r}^{(j)}\right]^{s}\left[-(n-1) \mathbf{Y}_{k}^{(j)}(\boldsymbol{\mu})\right]^{l-s}\right\} .
\end{aligned}
$$

Using the binomial theorem for times, the sth exponentiation in equation (27) is expressed as 


$$
\begin{aligned}
E\left\{\left[\sum_{r=1}^{n} \mathbf{Y}_{k, r}^{(j)}\right]^{s}\right\} & =E\left\{\sum_{k_{1}=0}^{s} C_{s}^{k_{1}}\left[\sum_{r=1}^{n-1} \mathbf{Y}_{k, r}^{(j)}\right]^{k_{1}}\left[\mathbf{Y}_{k, n}^{(j)}\right]^{s-k_{1}}\right\} \\
& =E\left\{\sum_{k_{1}=0}^{s} C_{s}^{k_{1}}\left\{\sum_{k_{2}=0}^{k_{1}} C_{k_{1}}^{k_{2}}\left[\sum_{r=1}^{n-2} \mathbf{Y}_{k, r}^{(j)}\right]^{k_{2}}\left[\mathbf{Y}_{k, n-1}^{(j)}\right]^{k_{1}-k_{2}}\right\}\left[\mathbf{Y}_{k, n}^{(j)}\right]^{s-k_{1}}\right\} \\
& =E\left\{\sum_{k_{1}=0}^{s} C_{s}^{k_{1}}\left\{\sum_{k_{2}=0}^{k_{1}} C_{k_{1}}^{k_{2}}\left\{\ldots \sum_{k_{n}=0}^{k_{n-1}} C_{k_{n-1}}^{k_{n}}\left[\mathbf{Y}_{k, 1}^{(j)}\right]^{k_{n}}\left[\mathbf{Y}_{k, 2}^{(j)}\right]^{k_{n-1}-k_{n}}\right\} \cdots\right\}\left[\mathbf{Y}_{k, n}^{(j)}\right]^{s-k_{1}}\right\} \\
& =\sum_{k_{1}=0}^{s} C_{s}^{k_{1}}\left\{\sum_{k_{2}=0}^{k_{1}} C_{k_{1}}^{k_{2}}\left\{\cdots \sum_{k_{n}=0}^{k_{n-1}} C_{k_{n-1}}^{k_{n}} E\left\{\left[\mathbf{Y}_{k, 1}^{(j)}\right]^{k_{n}}\right\} E\left\{\left[\mathbf{Y}_{k, 2}^{(j)}\right]^{k_{n-1}-k_{n}}\right\}\right\} \ldots\right\} E\left\{\left[\mathbf{Y}_{k, n}^{(j)}\right]^{s-k_{1}}\right\} .
\end{aligned}
$$

It is obvious from the above expression that when the origin moments of $\mathbf{Y}$ are obtained, the statistical moment of the random extreme response can be evaluated afterwards. As $Y_{k, r}^{(j)}$ is a function with only one random variable, it is expediently calculated by Gaussian numerical integration:

$$
E\left\{\left[Y_{k, r}^{(j)}\right]^{l}\right\}=\int_{-\infty}^{\infty}\left[Y_{k, r}^{(j)}\right]^{l} f_{u_{r}}\left(u_{r}\right) d u_{r}
$$

where $f_{u_{v}}$ is the probability density function of the standard normal variable $u_{r}$. The approach for estimating the statistical moment of the response function above is the socalled point estimate method, which is developed by Zhao [35].

Supposing $\mathbf{M}_{l}^{(j)}=E\left\{\left[\widehat{\mathbf{Y}}_{k}^{(j)}\right]^{l}\right\}(j=0,1, \ldots, N)$, the first four central moments of the random response can be derived from equations (30)-(33) as

$$
\begin{aligned}
E[\widehat{\mathbf{Y}}]= & E\left[\mathbf{Y}^{(0)}(t)\right]+E\left[\sum_{j=1}^{N} \xi_{j} \mathbf{Y}^{(j)}(t)\right] \\
= & E\left[\mathbf{Y}^{(0)}(t)\right]+\sum_{j=1}^{N} E\left[\xi_{j}\right] E\left[\mathbf{Y}^{(j)}(t)\right]=\mathbf{M}_{1}^{(0)} \\
\sigma^{2}[\widehat{\mathbf{Y}}]= & E\left\{\left[\mathbf{Y}-\mathbf{M}_{1}^{(0)}\right]^{2}\right\} \\
= & E\left\{\left[\mathbf{Y}^{(0)}(t)\right]^{2}\right\}+\sum_{j=1}^{N} E\left\{\left[\mathbf{Y}^{(j)}(t)\right]^{2}\right\} \\
& -\left[\mathbf{M}_{1}^{(0)}\right]^{2}=\mathbf{M}_{2}^{(0)}+\sum_{j=1}^{N} \mathbf{M}_{2}^{(j)}-\left[\mathbf{M}_{1}^{(0)}\right]^{2}, \\
\mathbf{V}_{3}[\widehat{\mathbf{Y}}]= & E\left\{\left[\mathbf{Y}-\mathbf{M}_{1}^{(0)}\right]^{3}\right\} \\
= & E\left\{\left[\mathbf{Y}^{(0)}(t)+\sum_{j=1}^{N} \xi_{j} \mathbf{Y}^{(j)}(t)-\mathbf{M}_{1}^{(0)}\right]^{3}\right\}=\mathbf{M}_{3}^{(0)} \\
& -3 \mathbf{M}_{2}^{(0)} \mathbf{M}_{1}^{(0)}+2\left[\mathbf{M}_{1}^{(0)}\right]^{3},
\end{aligned}
$$

$$
\begin{aligned}
\mathbf{V}_{3}[\widehat{\mathbf{Y}}]= & E\left\{\left[\mathbf{Y}-\mathbf{M}_{1}^{(0)}\right]^{4}\right\} \\
= & E\left\{\left[\mathbf{Y}^{(0)}(\mathrm{t})+\sum_{j=1}^{N} \xi_{j} \mathbf{Y}^{(j)}(t)-\mathbf{M}_{1}^{(0)}\right]^{4}\right\} \\
= & \mathbf{M}_{4}^{(0)}-4 \mathbf{M}_{3}^{(0)} \mathbf{M}_{1}^{(0)}+6 \mathbf{M}_{2}^{(0)}\left[\mathbf{M}_{1}^{(0)}\right]^{2}-3\left[\mathbf{M}_{1}^{(0)}\right]^{4} \\
& +12 \mathbf{M}_{2}^{(0)} \sum_{j=1}^{N} \mathbf{M}_{2}^{(j)}-6\left[\mathbf{M}_{1}^{(0)}\right]^{2} \sum_{j=1}^{N} \mathbf{M}_{2}^{(j)}+6 \sum_{j=1}^{N} \mathbf{M}_{4}^{(j)} \\
& +\sum_{i=1}^{N} \sum_{j=1, j \neq i}^{N} \mathbf{M}_{2}^{(i)} \mathbf{M}_{2}^{(j)} .
\end{aligned}
$$

\section{Reliability-Based Sensitivity Analysis for the Random Vibrating System}

Consider a system with state function $g(\mathbf{X})$, where the vector $\mathbf{X}$ represents the input random variables with a joint probability density function $f_{\mathbf{X}}(\mathbf{X})$. The probability of failure $P_{f}=P(g(\mathbf{X})<0)$ of the system is defined as

$$
P_{f}=\int \cdots \int_{g(\mathbf{X})<0} f_{\mathbf{X}}(\mathbf{X}) d \mathbf{X},
$$

where $g<0$ indicates the failure state, $g>0$ the safe state, and $g=0$ the limit state.

The effect of the distribution parameters on the reliability of the basic input variables is defined as the reliability-based sensitivity, which can be obtained by the methodology of derivation expressed as

$$
\frac{\partial P_{f}}{\partial \theta}=\frac{\partial \int \cdots \int_{g(\mathbf{X})<0} f_{\mathbf{X}}(\mathbf{X}, \boldsymbol{\theta}) d \mathbf{X}}{\partial \theta}=\int \cdots \int_{g(\mathbf{X})<0} \frac{\partial f_{\mathbf{X}}(\mathbf{X}, \boldsymbol{\theta})}{\partial \theta} d \mathbf{X},
$$

where $\boldsymbol{\theta}$ is the distribution parameter of the basic input variables such as the mean value and the variance, and the probability density function $f_{\mathbf{X}}(\mathbf{X})$ of $\mathbf{X}$ and $\boldsymbol{\theta}$ is expressed as $f_{\mathbf{X}}(\mathbf{X}, \boldsymbol{\theta})$. 
However, in many engineering systems, some input random variables may depend on time, and thus, the response can be described by a random process. In this case, a time-dependent analysis is necessary to reveal the reliability and reliability-based sensitivity characteristics of the random process system. Denoting the interested time interval as $\left[t_{\min }, t_{\max }\right]$, the instantaneous probability of failure is

$$
P_{f}\left(t_{\text {min }}, t_{\text {max }}\right)=P\left(g(\mathbf{X}, t)<0, \quad \exists t \in\left[t_{\text {min }}, t_{\text {max }}\right]\right),
$$

where the probability of failure is calculated in a quasi-steady sense, by fixing time $t$ and replacing the random process with a random variable.

In the numeric solving process, the time interval $\left[t_{\mathrm{min}}\right.$, $\left.t_{\text {max }}\right]$ is always dispersed to $q$ discrete points; the failure probability at each discrete point $t_{i}(i=1, \ldots, q)$ is defined by equation (34), which can be denoted as $P_{f i}=P\left[g\left(\mathbf{X}, t_{i}\right)<0\right]$; and the failure probability in the time interval is

$$
P_{f}\left(t_{\min }, t_{\max }\right)=\bigcup_{i=1}^{q} P_{f i},
$$

which requires to evaluate the union of failure value in all domains by solving $q$ performance functions acting together.

The limit-state function in the time-dependent problem can be written as

$$
g=\mathbf{G}-Y,
$$

where $G$ is the threshold value and $Y$ is the random response. The reliability can be investigated by the fourth-moment method [36].

The first four central moments of the limit-state function is as equations (39)-(42), under the assumption that $G$ and $Y$ are independent:

$$
\begin{aligned}
E[g] & =E[G]-E[Y], \\
\sigma^{2}[g] & =\sigma^{2}[G]+\sigma^{2}[Y], \\
V_{3}[g] & =V_{3}[G]-V_{3}[Y], \\
V_{4}[g] & =V_{4}[G]+V_{4}[Y]+6 \sigma^{2}[G] \sigma^{2}[Y],
\end{aligned}
$$

where $E(\cdot)$ indicates the mathematical expectation, $\sigma^{2}[\cdot]$ indicates the variance, $V_{3}[\cdot]$ denotes the third central moment, and $V_{4}[\cdot]$ is the fourth central moment, and the random response $Y$ can be substituted by $\widehat{Y}$.

The reliability index is defined as

$$
\beta_{2}=\frac{E[g]}{\sigma[g]} .
$$

The performance function $g$ can be standardized as

$$
z_{u}=\frac{g-E[g]}{\sigma[g]} .
$$

Let $\alpha_{3 g}=V_{3}[g] / \sigma^{3}[g]$ and $\alpha_{4 g}=V_{4}[g] / \sigma^{4}[g]$, which denote skewness and kurtosis of the state function, respectively. The Edgeworth series can be used to approximate the distribution function of the standardized variable $z_{u}$, which is expanded as

$$
\begin{aligned}
F\left(z_{u}\right)= & \Phi\left(z_{u}\right)-\phi\left(z_{u}\right)\left[\frac{1}{6} \alpha_{3 g} H_{2}\left(z_{u}\right)\right. \\
& \left.+\frac{1}{24}\left(\alpha_{4 g}-3\right) H_{3}\left(z_{u}\right)+\frac{1}{72} \alpha_{3 g}^{3} H_{5}\left(z_{u}\right)\right],
\end{aligned}
$$

where

$$
\begin{aligned}
& H_{2}(x)=x^{2}-1, \\
& H_{3}(x)=x^{3}-3 x, \\
& H_{5}(x)=x^{5}-10 x^{2}+15 x,
\end{aligned}
$$

are the second-, third-, and fifth-order Hermite polynomials, respectively.

The failure probability is

$$
P(g \leq 0)=P\left(z_{u} \leq \beta_{2}\right) .
$$

According to equation (45), the failure probability is expressed as

$$
\begin{aligned}
P= & F\left(-\beta_{2}\right)=\Phi\left(-\beta_{2}\right)-\phi\left(\beta_{2}\right) \\
& \cdot\left[\frac{1}{6} \alpha_{3 g} H_{2}\left(-\beta_{2}\right)+\frac{1}{24}\left(\alpha_{4 g}-3\right) H_{3}\left(-\beta_{2}\right)+\frac{1}{72} \alpha_{3 g}^{3} H_{5}\left(-\beta_{2}\right)\right],
\end{aligned}
$$

and the reliability is obtained as

$$
R=1-P=1-F\left(-\beta_{2}\right) .
$$

Because only the first four terms of the Edgeworth series are used, sometimes the reliability $R>1$ may occur. When $R$ is larger than 1, a revised formula is used to obtain the accurate solution [37]:

$$
R *\left(\beta_{2}\right) \approx \Phi\left(\beta_{2}\right)-\frac{\beta_{2}^{5}}{72} \alpha_{3 g}^{2} \phi\left(\beta_{2}\right) .
$$

The direct derivation method is used to evaluate the reliability-based sensitivities of the distribution parameters $\theta_{k}$ of the basic variables. Here, the distribution parameters include mean value and variance:

$$
\begin{aligned}
\frac{d R}{d \theta_{k}}= & \frac{\partial R}{\partial \beta_{2}}\left[\frac{\partial \beta_{2}}{\partial E(g)} \frac{\partial E(g)}{\partial E(\widehat{Y})} \frac{\partial E(\widehat{Y})}{\partial \theta_{k}}+\frac{\partial \beta_{2}}{\partial \sigma(g)} \frac{\partial \sigma(g)}{\partial \sigma(\widehat{Y})} \frac{\partial \sigma(\widehat{Y})}{\partial \theta_{k}}\right] \\
& +\frac{\partial R}{\partial \alpha_{3 g}}\left[\frac{\partial \alpha_{3 g}}{\partial V_{3}(g)} \frac{\partial V_{3}(g)}{\partial V_{3}(\widehat{Y})} \frac{\partial V_{3}(\widehat{Y})}{\partial \theta_{k}}+\frac{\partial \alpha_{3 g}}{\partial \sigma(g)} \frac{\partial \sigma(g)}{\partial \sigma(\widehat{Y})} \frac{\partial \sigma(\widehat{Y})}{\partial \theta_{k}}\right] \\
& +\frac{\partial R}{\partial \alpha_{4 g}}\left[\frac{\partial \alpha_{4 g}}{\partial V_{4}(g)} \frac{\partial V_{4}(g)}{\partial V_{4}(\widehat{Y})} \frac{\partial V_{4}(\widehat{Y})}{\partial \theta_{k}}+\frac{\partial \alpha_{4 g}}{\partial V_{4}(g)} \frac{\partial V_{4}(g)}{\partial \sigma(\widehat{Y})} \frac{\partial \sigma(\widehat{Y})}{\partial \theta_{k}}\right. \\
& \left.+\frac{\partial \alpha_{4 g}}{\partial \sigma(g)} \frac{\partial \sigma(g)}{\partial \sigma(\widehat{Y})} \frac{\partial \sigma(\widehat{Y})}{\partial \theta_{k}}\right] .
\end{aligned}
$$

Let $\partial \mathrm{V}_{l} / \partial \theta_{k}$ denote the partial derivative of the $l$ th center moment of $\widehat{Y}$ with respect to $\theta_{k}$, which can be calculated by the integral 


$$
\begin{aligned}
\frac{\partial V_{l}}{\partial \theta_{k}} & =\int_{-\infty}^{+\infty} \cdots \int_{-\infty}^{+\infty}[\widehat{Y}]^{l} \frac{\partial f_{\mathbf{X}}(\mathbf{X}, \boldsymbol{\theta})}{\partial \theta_{k}} d \mathbf{X} \\
& =E\left\{\frac{[\widehat{Y}]^{l}}{f_{\mathbf{X}}(\mathbf{X}, \boldsymbol{\theta})} \frac{\partial f_{\mathbf{X}}(\mathbf{X}, \boldsymbol{\theta})}{\partial \theta_{k}}\right\}=E\left\{[\widehat{Y}]^{l} C_{\theta}\right\},
\end{aligned}
$$

where $C_{\boldsymbol{\theta}}=\left(1 / f_{\mathbf{X}}(\mathbf{X}, \boldsymbol{\theta})\right)\left(\partial f_{\mathbf{X}}(\mathbf{X}, \boldsymbol{\theta}) / \partial \theta_{k}\right)$ is the kernel function, and equation (52) can be calculated by numerical integration together with equation (29).

\section{Numerical Example}

5.1. Reliability and Reliability-Based Sensitivity Analysis of a Multi-Degree-of-Freedom Truss Structure. A truss structure is considered in this example, as shown in Figure 2, with parameters depicted in Table 1.

The stimulating force $F(t)$ is supposed to be a stochastic process with constant autospectrum $2 \times 10^{5} \mathrm{~N}^{2} / \mathrm{Hz}$, frequency domain $0-180 \mathrm{~Hz}$, and mean value $5 \times 10^{6} \mathrm{~N}$, and the power spectral density function and covariance function are as follows:

$$
\begin{aligned}
& S_{f f}(\omega)=S_{0} \frac{1+4 \eta_{g}^{2}\left(\omega / \omega_{g}\right)^{2}}{\left[1-\left(\omega / \omega_{g}\right)^{2}\right]^{2}+4 \eta_{g}^{2}\left(\omega / \omega_{g}\right)^{2}}, \\
& C_{f f}(\tau)=\frac{\pi S_{0}}{2} \mathrm{e}^{\eta_{g} \omega_{g}|\tau|}\left[\eta_{1} \cos \left(\omega_{d} \tau\right)+\eta_{2} \sin \left(\omega_{d}\right)|\tau|\right],
\end{aligned}
$$

where $\omega_{d}=\omega_{g} \sqrt{1-\eta_{g}^{2}}, \quad \eta_{1}=\omega_{g}\left(\left(1+4 \eta_{g}^{2}\right) / \eta_{g}\right), \quad \eta_{2}=\omega_{g}$ $\left(\left(1-4 \eta_{g}^{2}\right) /\left(1-\eta_{g}^{2}\right)\right), \quad \omega_{g}=1.5 \mathrm{rad} / \mathrm{s}, \quad \eta_{g}=1.2$, and $S_{0}=$ $1 \mathrm{e} 5 \mathrm{~m}^{2} / \mathrm{s}^{3}$.

When $\tau$ equals 0 , the variance of the random process $\sigma^{2}(t)=\pi S_{0} / 2$.

First, the stiffness matrix, mass matrix, and damping matrix are established by the FEM, and then the vibration equation of this truss is developed. The vertical response at node 4 is considered for reliability analysis. Rosenblatt transformation is used here to transfer the lognormal normal distribution parameters to independent normal distribution parameters. Then, the proposed method and the MCS are constructed to estimate the reliability and reliability-based sensitivity of the parameters of the structure. The number of $\mathrm{K}-\mathrm{L}$ expansion terms is selected to be 300 , and the tolerance values $\varepsilon_{\mathrm{Var}, N}$ and $\varepsilon_{\mathrm{Cov}, N}$ are about $10^{-15}$ and $10^{-10}$. They are accurate enough for calculation. The 100 realizations of random exciting force are simulated in Figure 3. The 100 realizations of response of the structure are shown in Figure 4.

The transient reliability response curve is shown in Figure 5, and the accumulative reliability response curve is shown in Figure 6. The results obtained from the MCS method and the method proposed in this paper agree very well with each other, which indicates that the method proposed here has great computing accuracy and computing efficiency. The transient reliability curve shows that the reliability is minimum at $0.04 \mathrm{~s}$ after start, so it is the most dangerous time for the system. The transient curve and the accumulative curve are kept stable after the dangerous time. In the stable state, the reliability-based sensitivity of the

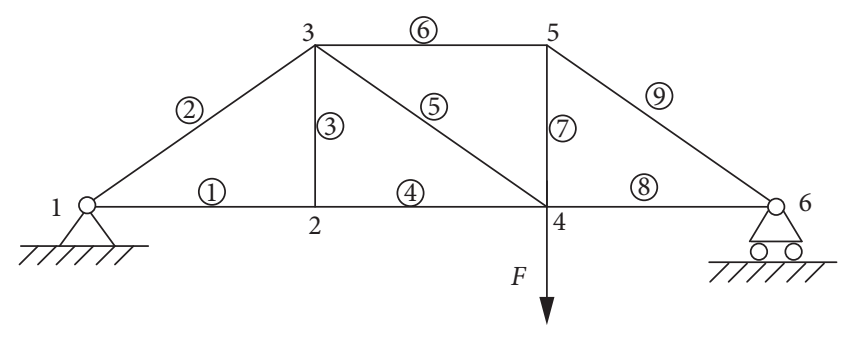

Figure 2: Model of truss.

TABle 1: Parameters of the truss structure.

\begin{tabular}{lcc}
\hline Parameters & Mean value & Distribution style \\
\hline Cross-sectional area & $2.5 \times 10^{-3} \mathrm{~m}^{2}$ & Lognormal distribution \\
Elastic modulus of & $2.0 \times 10^{11} \mathrm{pa}$ & Lognormal distribution \\
materials & $7.86 \times 10^{3} \mathrm{~kg} / \mathrm{m}^{3}$ & Lognormal distribution \\
Density & & \\
\hline
\end{tabular}

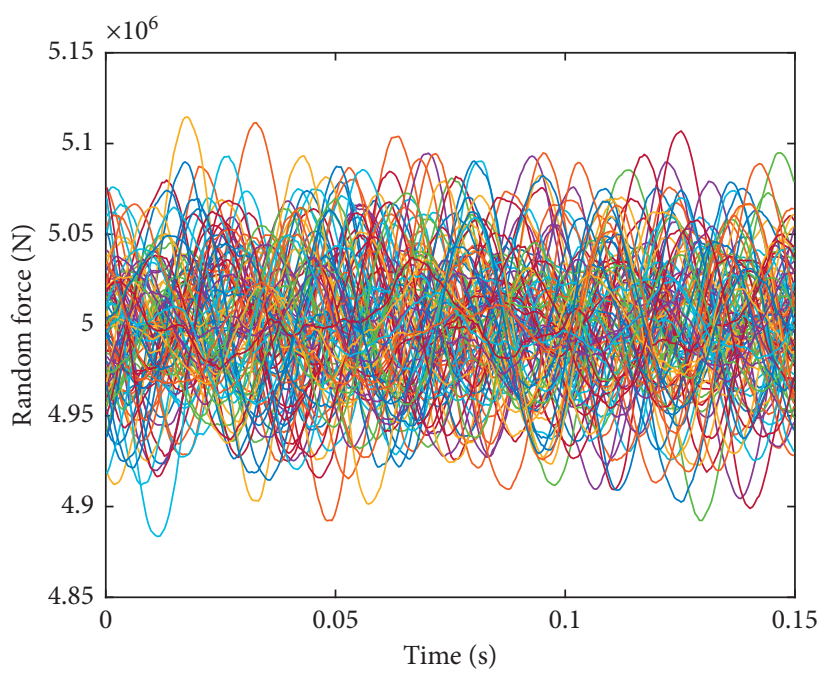

FIGURE 3: 100 realizations of random exciting force.

parameters is obtained, as shown in Figure 7, and the positive influence of the mean value of the cross-sectional area and the negative influence of the variance of the crosssectional area are outstanding.

5.2. System with Unknown Distribution Parameters. A damped dynamic vibration absorber model is shown in Figure 8, which is used as the vibrating screen for instance. As illustrated in Figure 8, the system is composed of a vibrating system (mass $m_{1}$, spring $k_{1}$, and viscous damping $c_{1}$ ) and a dynamic shock absorption system (mass $m_{2}$, spring $k_{2}$, and viscous damping $c_{2}$ ), and the statistical moments of the random parameters of the system with unknown distributions are displayed in Table 2.

The different equation of motion is

$$
\begin{aligned}
& m_{1} \ddot{x}_{1}+\left(k_{1}+k_{1}\right) x_{1}-k_{2} x_{2}+\left(c_{1}+c_{2}\right) \dot{x}_{1}-c_{2} \dot{x}_{2} \\
& \quad=F_{1} \sin \left(\omega_{t}\right)+A(t) f(t), \\
& m_{2} \ddot{x}_{2}-k_{2} x_{1}+k_{2} x_{2}-c_{2} \dot{x}_{1}+c_{2} \dot{x}_{2}=0 .
\end{aligned}
$$




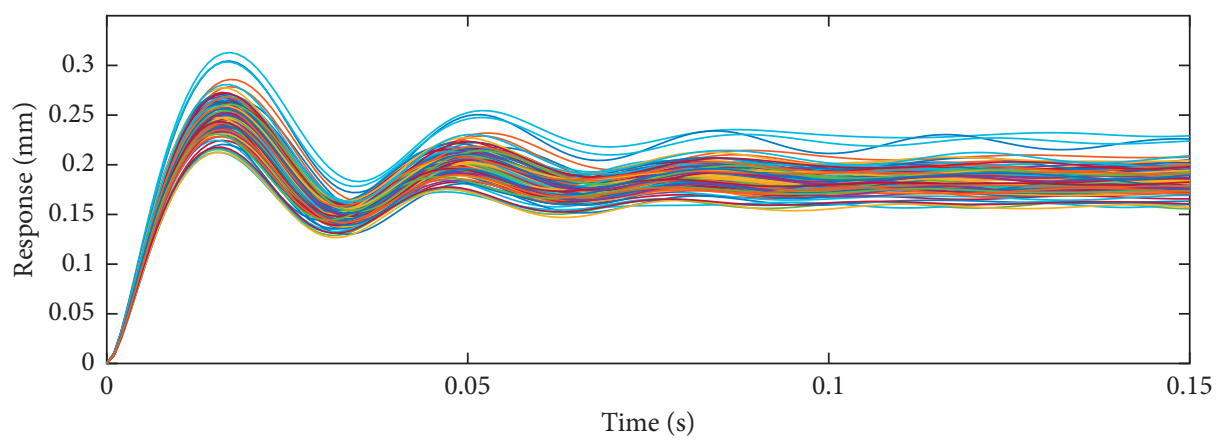

FIgURE 4: 100 realizations of vertical response at node 4 .

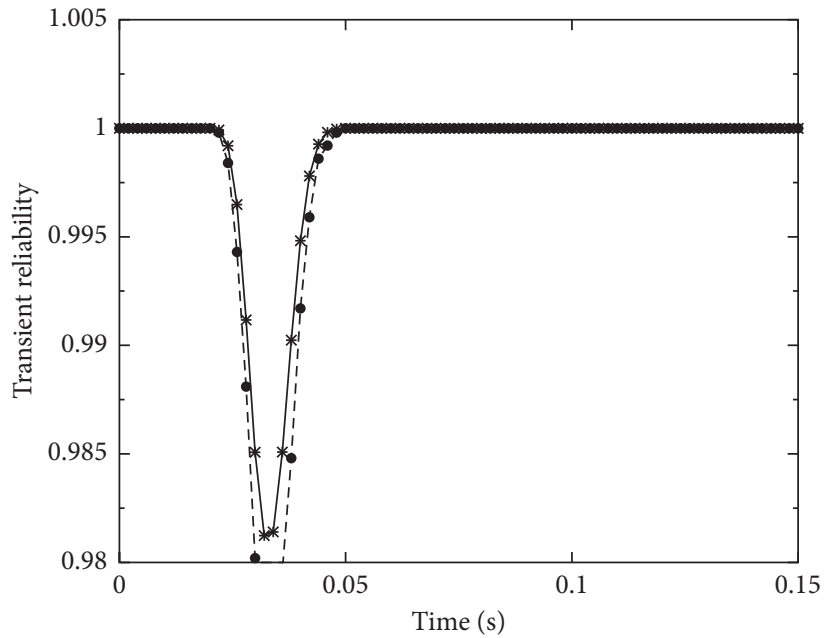

$\rightarrow$ Proposed method

-• MCS

FIgURE 5: Transient reliability curve of response.

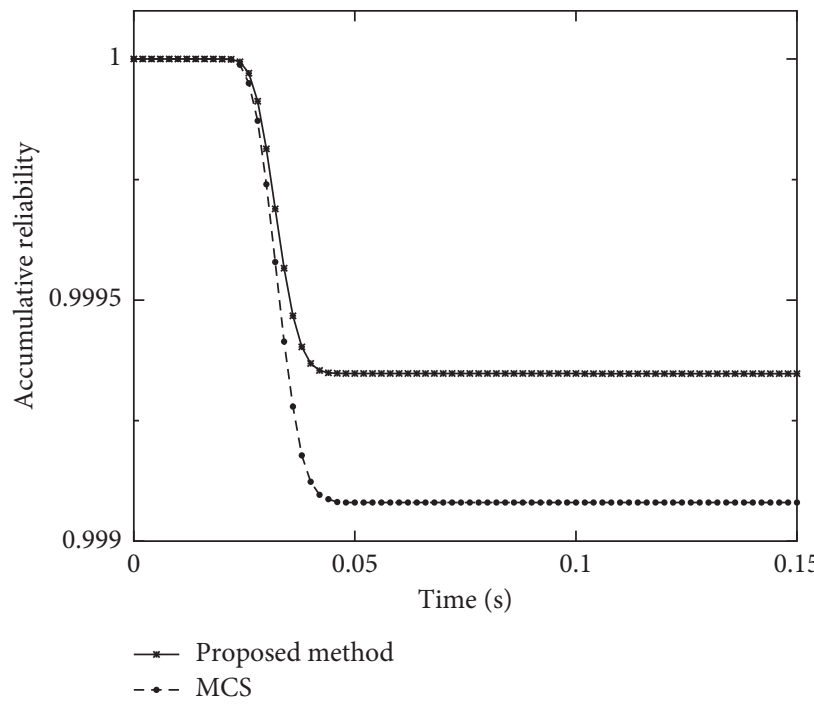

FIgURE 6: Accumulative reliability curve of response. 

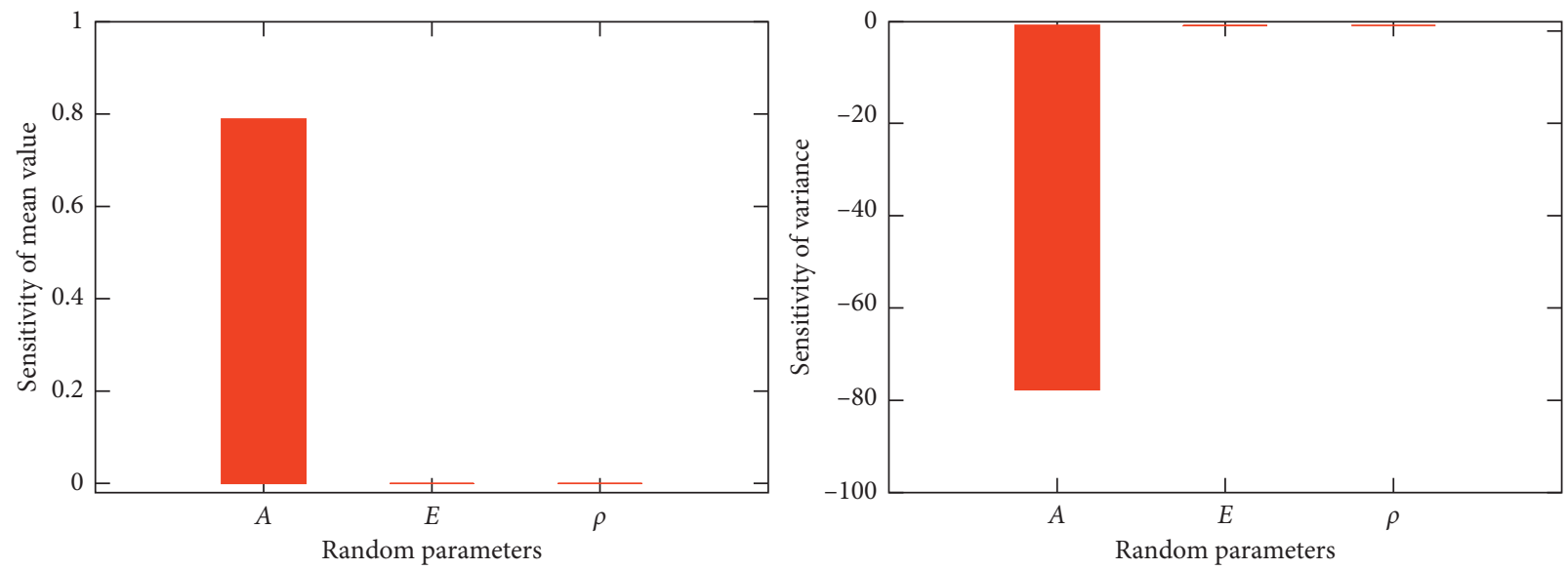

FIgURE 7: Reliability-based sensitivity of the random parameters.

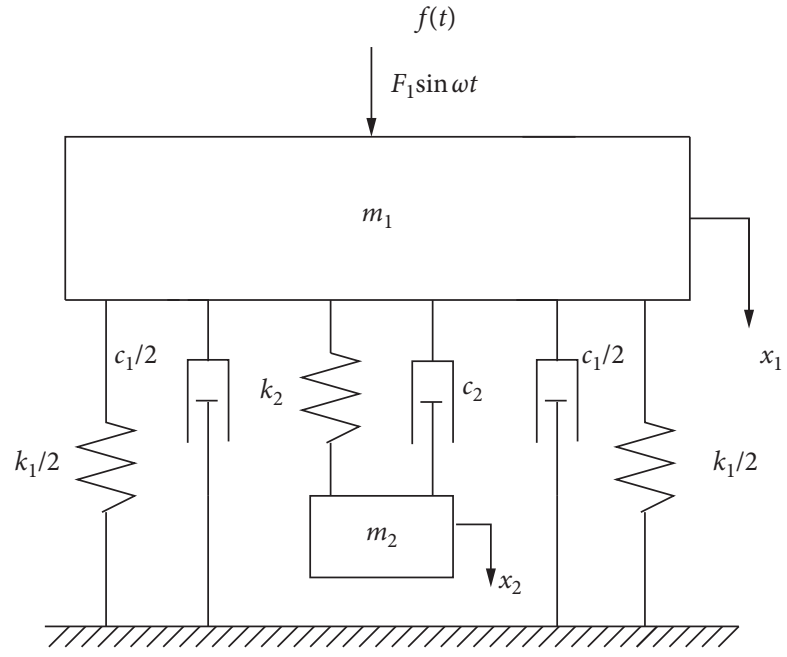

Figure 8: Dynamic absorber model.

TABLE 2: Stochastic characteristics of basic parameters.

\begin{tabular}{|c|c|c|c|c|c|c|c|c|}
\hline Random parameters & $m_{1}(\mathrm{~kg})$ & $k_{1}(\mathrm{~N} / \mathrm{m})$ & $c_{1}(\mathrm{~N} \cdot \mathrm{s} / \mathrm{m})$ & $m_{2}(\mathrm{~kg})$ & $k_{2}(\mathrm{~N} / \mathrm{m})$ & $c_{2}(\mathrm{~N} \cdot \mathrm{s} / \mathrm{m})$ & $\omega_{t}(\mathrm{rad} / \mathrm{s})$ & $F_{1}(\mathrm{~N})$ \\
\hline Average & 500 & $1 \mathrm{e} 6$ & $1 \mathrm{e} 5$ & 50 & $1 \mathrm{e} 7$ & $1 \mathrm{e} 5$ & 50 & 8000 \\
\hline Variance & 25 & $5 \mathrm{e} 4$ & $5 \mathrm{e} 3$ & 2.5 & $5 \mathrm{e} 5$ & $5 \mathrm{e} 3$ & 2.5 & $4 \mathrm{e} 2$ \\
\hline Third moment & $2.55 \mathrm{e} 3$ & $2.10 \mathrm{e} 3$ & $2.21 \mathrm{e} 6$ & 3.26 & $2.32 \mathrm{e} 6$ & $2.21 \mathrm{e} 6$ & 1.6387 & $6.67 \mathrm{e} 6$ \\
\hline Fourth moment & $1.22 \mathrm{e} 6$ & $1.96 \mathrm{e} 19$ & $1.13 \mathrm{e} 10$ & 120.26 & $1.98 \mathrm{e} 23$ & $1.13 \mathrm{e} 10$ & 121.75 & 7.85 \\
\hline
\end{tabular}

The stimulating force $f(t)$ is the stochastic disturbance in work, which is a uniform modulated nonstationary stochastic process. The modulating function is

$$
A(t)=\left\{\begin{array}{lr}
\frac{t^{2}}{t_{b}^{2}}, & 0 \leq t \leq t_{b}, \\
1.0, & t_{b} \leq t \leq t_{c}, \\
e^{-c\left(t-t_{c}\right)}, & t \geq t_{c},
\end{array}\right.
$$

where $t_{b}=3 \mathrm{~s}, t_{c}=6 \mathrm{~s}$, and $c=0.1572$. The power spectral density function and the covariance function of a Gaussian stochastic process are expressed as equations (53) and (54), respectively, in which $\omega_{g}=15 \mathrm{rad} / \mathrm{s}, \quad \eta_{g}=0.06$, and
$S_{0}=1 \mathrm{e} 5 \mathrm{~m}^{2} / \mathrm{s}^{3}$. Considering $\tau=t_{2}-t_{1}$, the power spectral density function and covariance of the modulated process are

$$
\begin{aligned}
S_{f f}(\omega)= & A(t)^{2} S_{0} \frac{1+4 \eta_{g}^{2}\left(\omega / \omega_{g}\right)^{2}}{\left[1-\left(\omega / \omega_{g}\right)^{2}\right]^{2}+4 \eta_{g}^{2}\left(\omega / \omega_{g}\right)^{2}}, \\
C_{f f}(\tau)= & A\left(t_{1}\right) A\left(t_{2}\right) \frac{\pi S_{0}}{2} e^{\eta_{g} \omega_{g}|\tau|} \\
& \cdot\left[\eta_{1} \cos \left(\omega_{d} \tau\right)+\eta_{2} \sin \left(\omega_{d}\right)|\tau|\right] .
\end{aligned}
$$

The number of K-L expansion terms is selected to be 200, and the tolerance values $\varepsilon_{\mathrm{Var}, \mathrm{N}}$ and $\varepsilon_{\mathrm{Cov}, \mathrm{N}}$ are about $10^{-13}$ and 


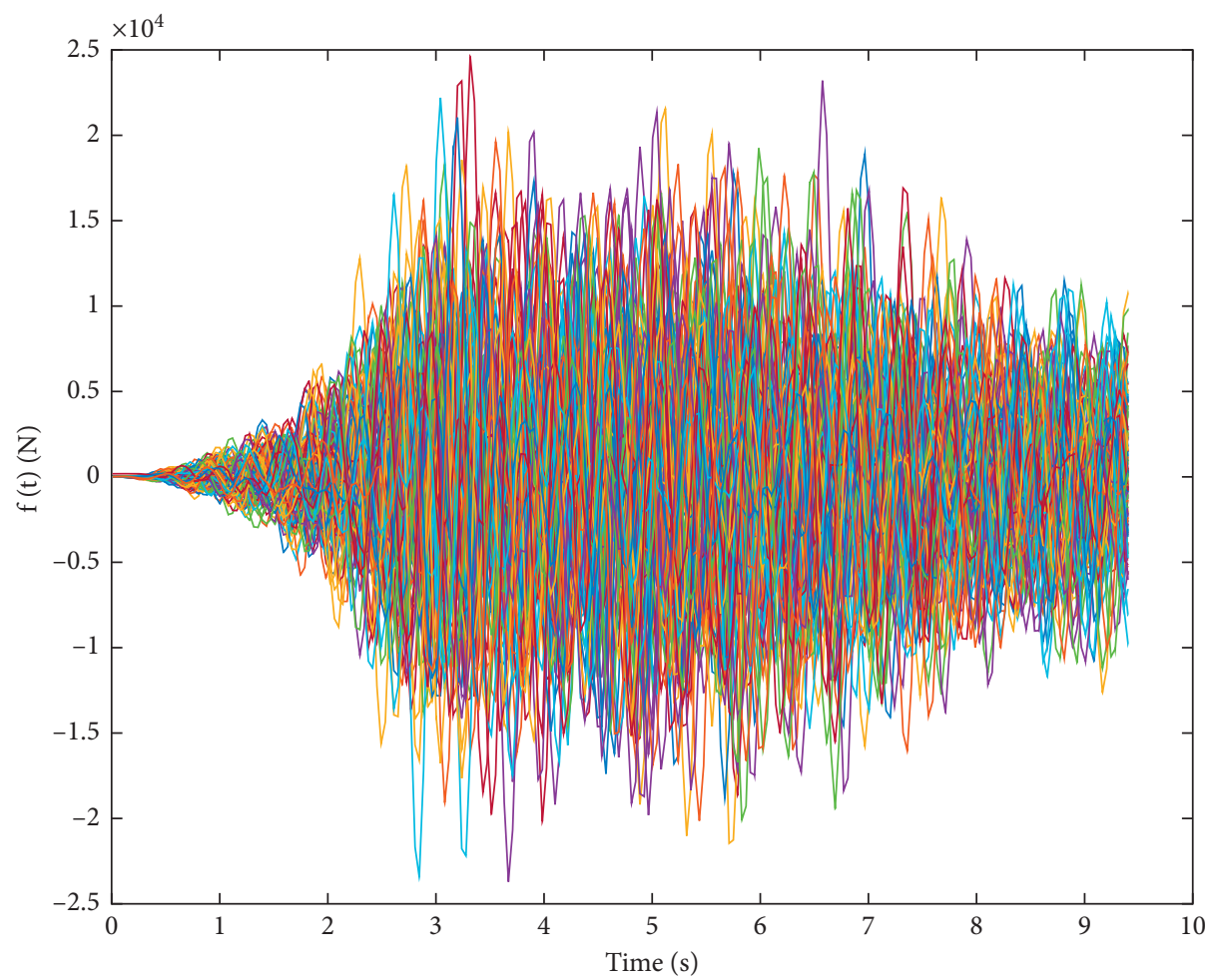

FIgURE 9: 100 realizations of the exciting force.

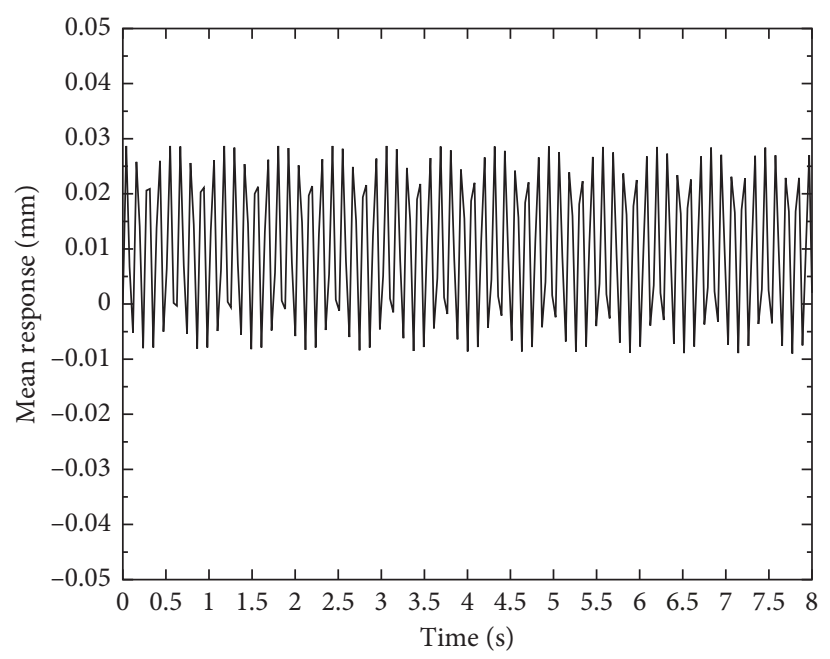

Figure 10: Mean response of the vibration absorber model.

$10^{-8}$, which are accurate enough. The 100 realizations of the random exciting force are shown in Figure 9. The mean response of the structure is shown in Figure 10. The perturbation method proposed in paper [23] is used here to solve the problem in the system with unknown distribution random parameters. Given the first four moments of the random parameters, the statistical characteristic of the response can be calculated. And then, the reliability and reliability-based sensitivity can be obtained. The transient reliability and the accumulative reliability obtained by the method proposed in this paper comparing with the MCS method are shown in Figures 11 and 12. Different from the examples proposed above, the curves of the reliability cannot reach a stable state because of the system's unstable characteristic.

To investigate the influence of the random parameters on the reliability, the accumulative sensitivities are calculated which are shown in Figure 13. According to Figure 13, the mean values of $m_{1}, k_{1}$, and $\omega_{\mathrm{t}}$ have a positive effect on the reliability significantly, while the mean value of $c_{2}$ and the variance of $m_{2}, k_{2}, c_{1}$, and $\omega_{\mathrm{t}}$ have a negative effect on the reliability significantly. 


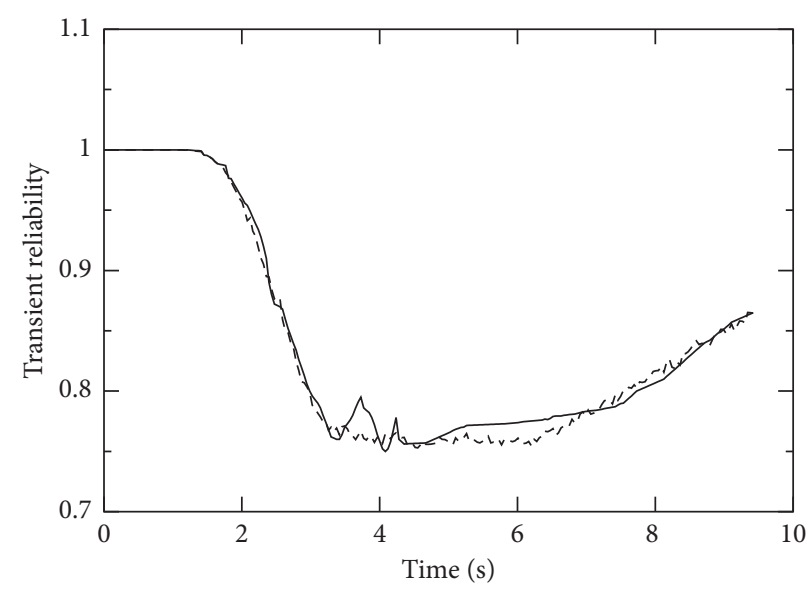

— Proposed method

- - MCS

Figure 11: Transient reliability curve of response.

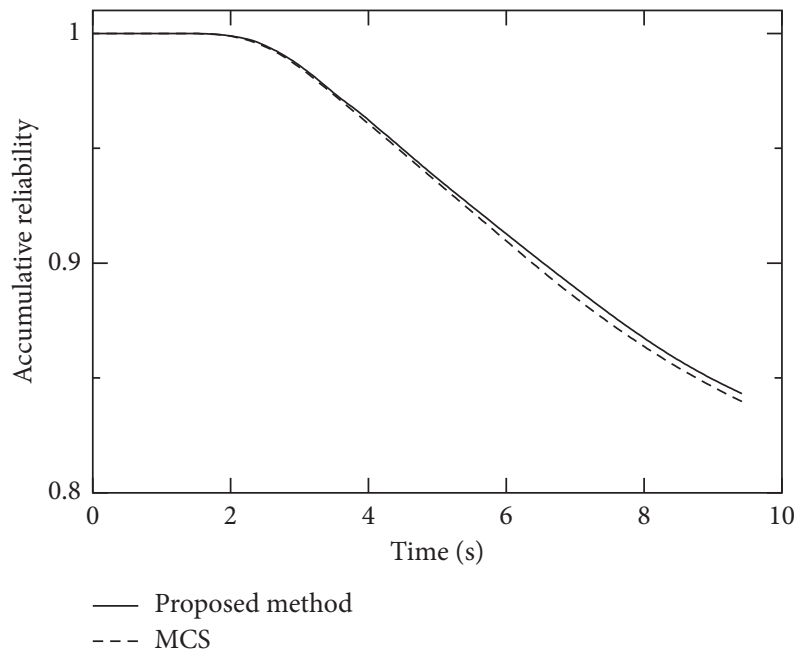

FIGURE 12: Accumulative reliability curve of response.
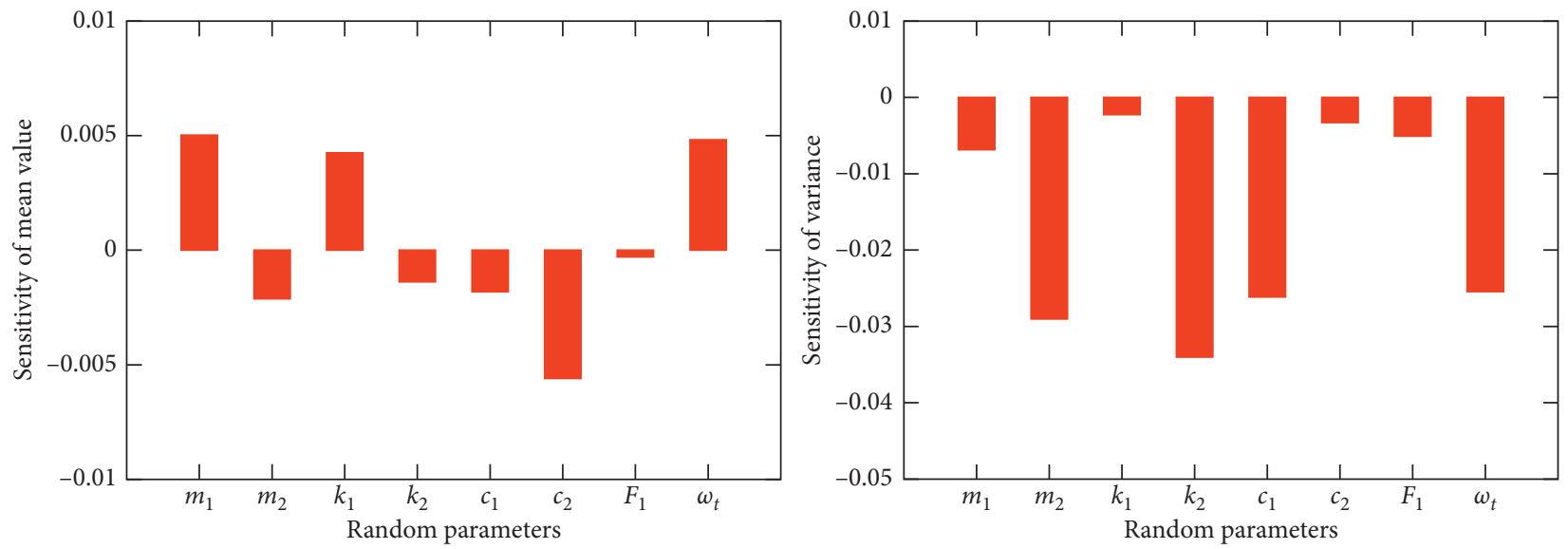

FIGURE 13: Reliability-based sensitivity of the random parameters. 


\section{Conclusion}

For the random parametric systems subjected to random process excitation, a method combining the stochastic process K-L expansion method, the precise Gauss-Legendre integration, the point estimate method, and the fourthmoment method is proposed in this paper. The precise integration is suitable for solving differential functions in a time-saving way and can combine with the K-L expansion method at the same dispersed time points. The combination of the two methods simplifies the calculation process and makes it realizable for estimate structure responses subjected to a large number of expansion exciting forces. A large number of Gaussian random parameters produced in the K-L expansion scarcely contributed to difficulties in the moment calculation according to equations (30)-(33). However, the method has its own deficiencies in the structure model, which is required to be linear to apply the principle of superposition.

Therefore, the method proposed in this paper is recommended to be applied in the reliability problems of linear structures with time-independent and time-dependent random parameters, which is easy to implement and has high efficiency and accuracy.

\section{Data Availability}

The result data used to support the findings of this study are available from the corresponding author upon request.

\section{Conflicts of Interest}

The authors declare that they have no conflicts of interest.

\section{Acknowledgments}

The authors gratefully acknowledge the Shandong Provincial Natural Science Foundation, China (ZR2014EEP015), and Doctoral Science Foundation of Weifang University.

\section{References}

[1] A. Chaudhuri and S. Chakraborty, "Reliability of linear structures with parameter uncertainty under non-stationary earthquake," Structural Safety, vol. 28, no. 3, pp. 231-246, 2006.

[2] J.-B. Chen and J. Li, "Dynamic response and reliability analysis of non-linear stochastic structures," Probabilistic Engineering Mechanics, vol. 20, no. 1, pp. 33-44, 2005.

[3] S. Gupta and C. S. Manohar, "Reliability analysis of randomly vibrating structures with parameter uncertainties," Journal of Sound and Vibration, vol. 297, no. 3-5, pp. 1000-1024, 2006.

[4] P. Kumar and S. Narayanan, "Nonlinear stochastic dynamics, chaos and reliability analysis for single degree freedom model of a rotor blade," in Proceedings of the Asme Turbo Expo 2008, vol. 5, pp. 1341-1350, Berlin, Germany, June 2008.

[5] M. Papadrakakis and N. D. Lagaros, "Reliability-based structural optimization using neural networks and Monte Carlo simulation," Computer Methods in Applied Mechanics and Engineering, vol. 191, no. 32, pp. 3491-3507, 2002.
[6] S. Adhikari, "Sensitivity based reduced approaches for structural reliability analysis," Sadhana, vol. 35, no. 3, pp. 319-339, 2010.

[7] J.-Y. Chen, Q. Xu, J. Li, and S.-L. Fan, "Improved response surface method for anti-slide reliability analysis of gravity dam based on weighted regression," Journal of Zhejiang University-Science A, vol. 11, no. 6, pp. 432-439, 2010.

[8] H. Guimarães, J. C. Matos, and A. A. Henriques, "An innovative adaptive sparse response surface method for structural reliability analysis," Structural Safety, vol. 73, pp. 12-28, 2018.

[9] D. Zhou, X. Zhang, and Y. Zhang, "Reliability analysis of the traction unit of a shearer mechanism with response surface method," Journal of Mechanical Science and Technology, vol. 31, no. 10, pp. 4679-4689, 2017.

[10] A. A. Chojaczyk, A. P. Teixeira, L. C. Neves, J. B. Cardoso, and C. G. Soares, "Review and application of artificial neural networks models in reliability analysis of steel structures," Structural Safety, vol. 52, pp. 78-89, 2015.

[11] X. Zhang, M. D. Pandey, and Y. Zhang, "A numerical method for structural uncertainty response computation," Science China Technological Sciences, vol. 54, no. 12, pp. 3347-3357, 2011.

[12] X. F. Zhang, Y. E. Zhao, Y. M. Zhang, X. Z. Huang, and H. Li, "A points estimation and series approximation method for uncertainty analysis," Proceedings of the Institution of Mechanical Engineers, Part C: Journal of Mechanical Engineering Science, vol. 223, no. 9, pp. 1997-2007, 2009.

[13] S. Rahman, "Decomposition methods for structural reliability analysis revisited," Probabilistic Engineering Mechanics, vol. 26, no. 2, pp. 357-363, 2011.

[14] S. Rahman, "Wiener-Hermite polynomial expansion for multivariate Gaussian probability measures," Journal of Mathematical Analysis and Applications, vol. 454, no. 1, pp. 303-334, 2017.

[15] H. Xu and S. Rahman, "Decomposition methods for structural reliability analysis," Probabilistic Engineering Mechanics, vol. 20, no. 3, pp. 239-250, 2005.

[16] Z. Hu and S. Mahadevan, "A single-loop kriging surrogate modeling for time-dependent reliability analysis," Journal of Mechanical Design, vol. 138, no. 6, Article ID 061406, 2016.

[17] Z. Hu and X. Du, "Mixed efficient global optimization for time-dependent reliability analysis," Journal of Mechanical Design, vol. 137, no. 5, Article ID 051401, 2015.

[18] L. Wang, X. Zhang, and Y. Zhou, "An effective approach for kinematic reliability analysis of steering mechanisms," Reliability Engineering \& System Safety, vol. 180, pp. 62-76, 2018.

[19] H. J. Pradlwarter and G. I. Schuëller, "Excursion probabilities of non-linear systems," International Journal of Non-Linear Mechanics, vol. 39, no. 9, pp. 1447-1452, 2004.

[20] G. I. Schueller, H. J. Pradlwarter, and C. A. Schenk, "Nonstationary response of large linear FE models under stochastic loading," Computers \& Structures, vol. 81, no. 8-11, pp. 937-947, 2003.

[21] H. J. Pradlwarter and G. I. Schueller, "Uncertain linear structural systems in dynamics: efficient stochastic reliability assessment," Computers \& Structures, vol. 88, no. 1-2, pp. 74-86, 2010.

[22] H. J. Pradlwarter and G. I. Schuëller, "Reliability assessment of uncertain linear systems in structural dynamics," in Iutam Symposium on the Vibration Analysis of Structures with Uncertainties, A. K. Belyaev and R. S. Langley, Eds., vol. 27, pp. 363-378, Springer, Berlin, Germany, 2011. 
[23] Y. Zhang, "Perturbation method for reliability-based sensitivity analysis," in Proceedings of the ICMIT 2009: Mechatronics and Information Technology, J. Ryu, K. T. Chong, R. Ikeura, and Q. Han, Eds., Gwangju, South Korea, January 2010.

[24] S. Gupta and C. S. Manohar, "Improved response surface method for time-variant reliability analysis of nonlinear random structures under non-stationary excitations," Nonlinear Dynamics, vol. 36, no. 2-4, pp. 267-280, 2004.

[25] S. Gupta and C. S. Manohar, "An improved response surface method for the determination of failure probability and importance measures," Structural Safety, vol. 26, no. 2, pp. 123-139, 2004.

[26] D. V. Iourtchenko, E. Mo, and A. Naess, "Response probability density functions of strongly non-linear systems by the path integration method," International Journal of NonLinear Mechanics, vol. 41, no. 5, pp. 693-705, 2006.

[27] A. Naess and V. Moe, "Efficient path integration methods for nonlinear dynamic systems," Probabilistic Engineering $\mathrm{Me}$ chanics, vol. 15, no. 2, pp. 221-231, 2000.

[28] W. Betz, I. Papaioannou, and D. Straub, "Numerical methods for the discretization of random fields by means of the Karhunen-Loève expansion," Computer Methods in Applied Mechanics and Engineering, vol. 271, pp. 109-129, 2014.

[29] X. Zhang, Q. Liu, and H. Huang, "Numerical simulation of random fields with a high-order polynomial based RitzGalerkin approach," Probabilistic Engineering Mechanics, vol. 55, pp. 17-27, 2019.

[30] M.-H. Fu, M.-C. Cheung, and S. V. Sheshenin, "Precise integration method for solving singular perturbation problems," Applied Mathematics and Mechanics, vol. 31, no. 11, pp. 1463-1472, 2010.

[31] Q. Liu, J. Zhang, and L. Yan, "A numerical method of calculating first and second derivatives of dynamic response based on Gauss precise time step integration method," $E u$ ropean Journal of Mechanics-A/Solids, vol. 29, no. 3, pp. 370-377, 2010.

[32] M.-F. Wang and F. T. K. Au, "Precise integration methods based on Lagrange piecewise interpolation polynomials," International Journal for Numerical Methods in Engineering, vol. 77, no. 7, pp. 998-1014, 2009.

[33] Z. Wan-Xie, "On precise integration method," Journal of Computational and Applied Mathematics, vol. 163, no. 1, pp. 59-78, 2004.

[34] Z.-H. Lu, C.-H. Cai, and Y.-G. Zhao, "Structural reliability analysis including correlated random variables based on third-moment transformation," Journal of Structural Engineering, vol. 143, no. 8, Article ID 04017067, 2017.

[35] Y.-G. Zhao and T. Ono, "New point estimates for probability moments," Journal of Engineering Mechanics, vol. 126, no. 4, pp. 433-436, 2000.

[36] Y.-G. Zhao and T. Ono, "Moment methods for structural reliability," Structural Safety, vol. 23, no. 1, pp. 47-75, 2001.

[37] Y. Zhao and Y. Zhang, "Reliability design and sensitivity analysis of cylindrical worm pairs," Mechanism and Machine Theory, vol. 82, pp. 218-230, 2014. 


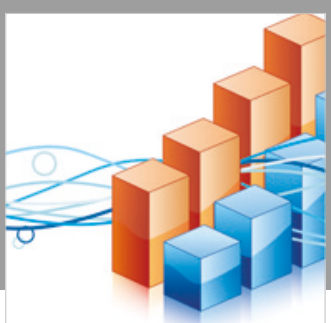

Advances in

Operations Research

\section{-n-m}
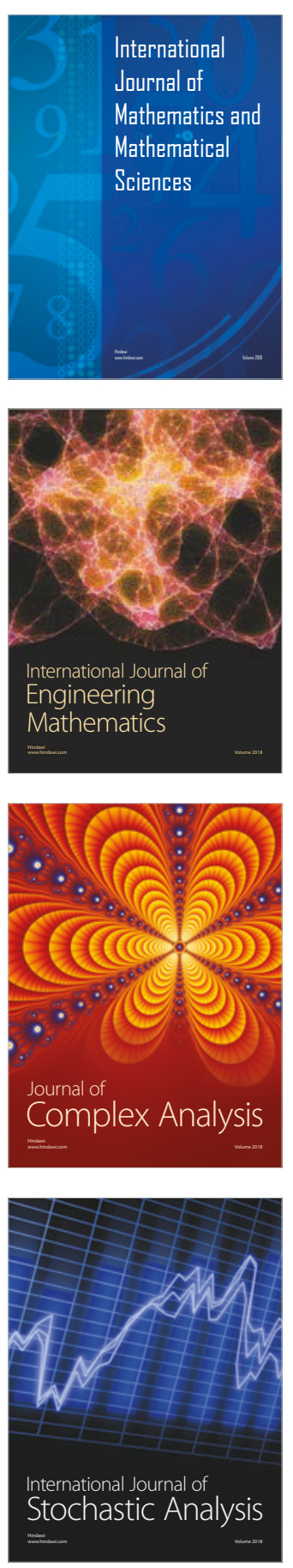
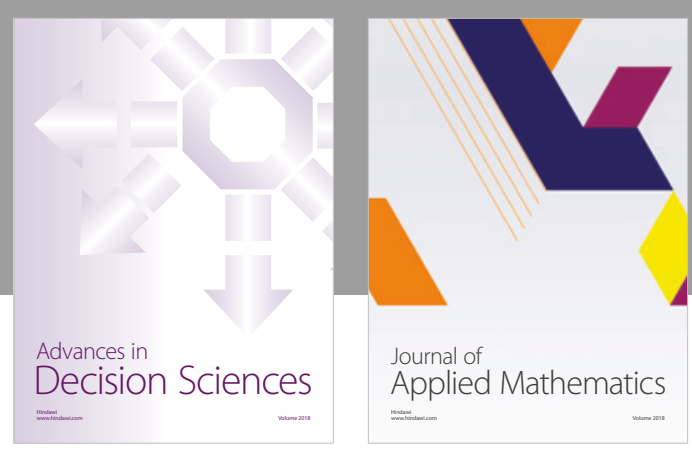

Journal of

Applied Mathematics
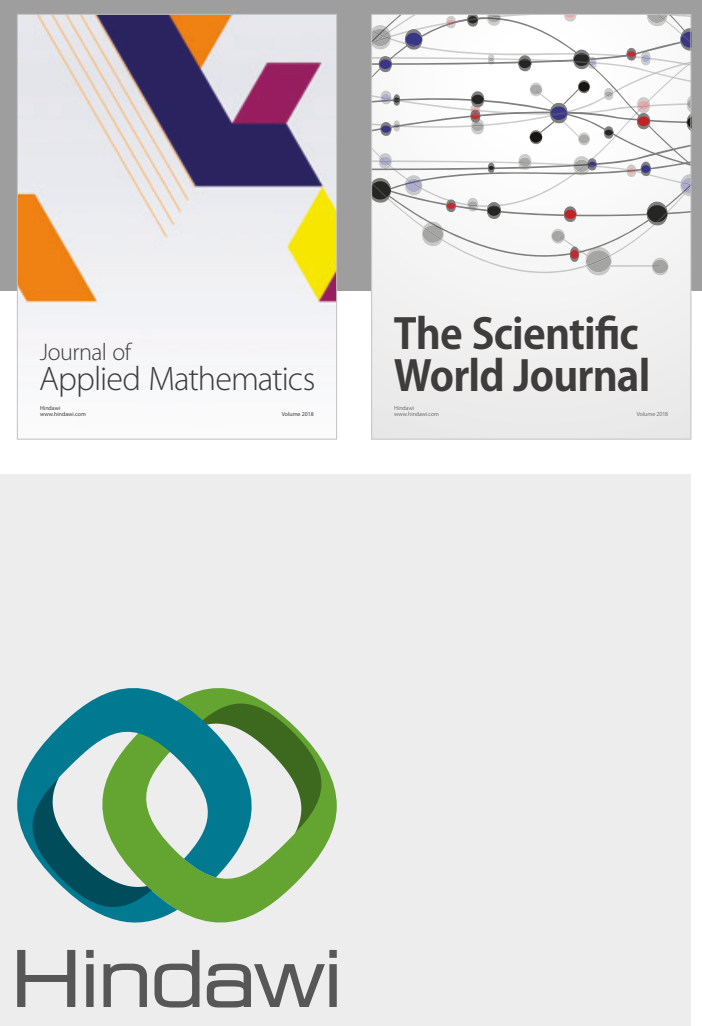

Submit your manuscripts at

www.hindawi.com

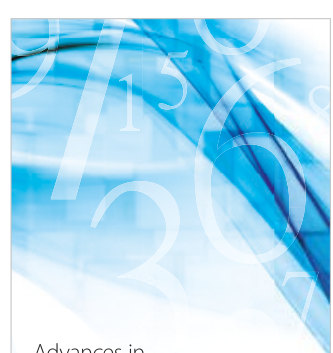

Advances in
Numerical Analysis
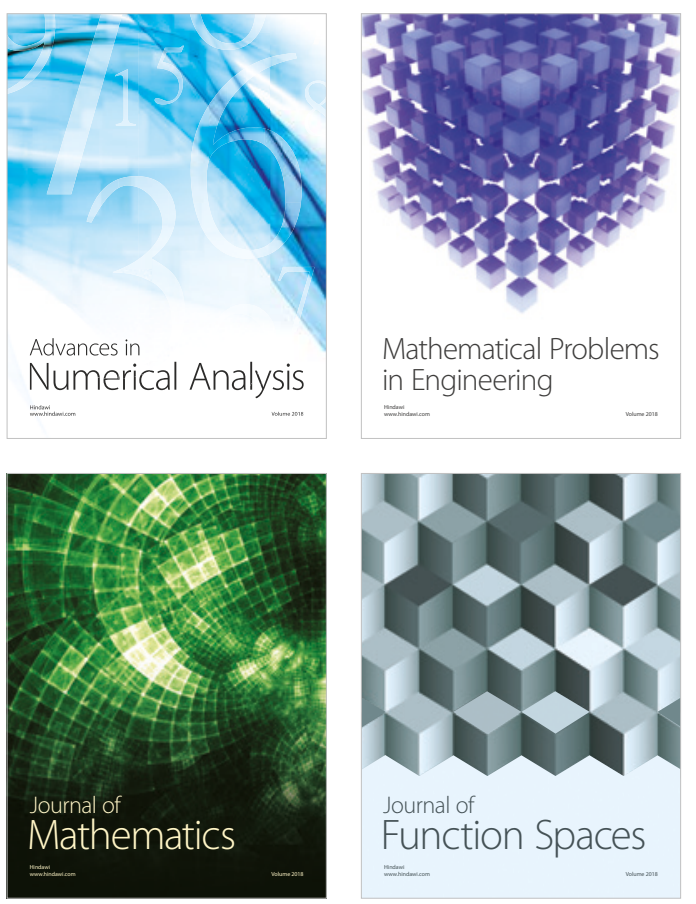

Mathematical Problems in Engineering

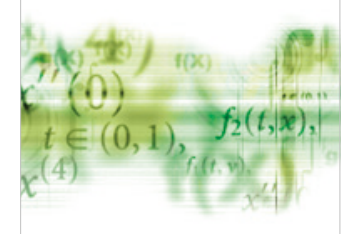

International Journal of

Differential Equations

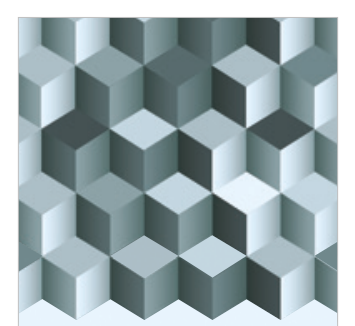

Journal of

Function Spaces

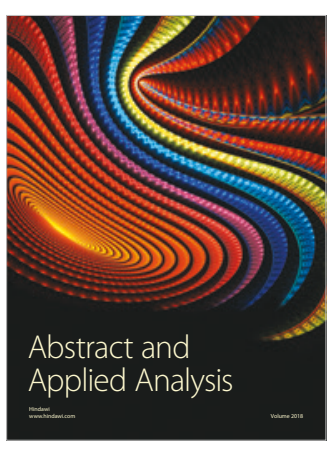

The Scientific

World Journal

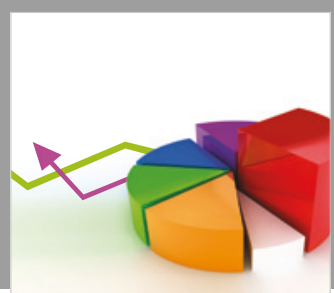

Journal of

Probability and Statistics
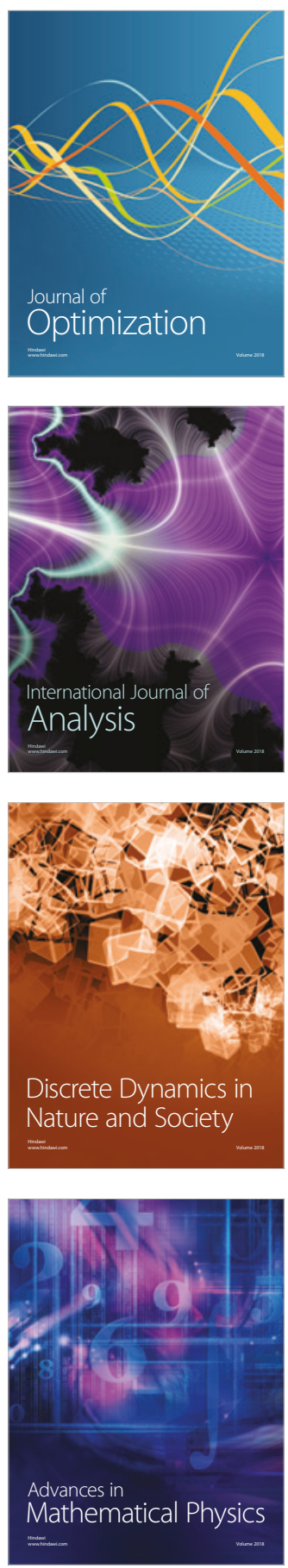